\title{
Erratum to: The cloud radiative effect on the atmospheric energy budget and global mean precipitation
}

\author{
F. Hugo Lambert • Mark J. Webb • \\ Masakazu Yoshimori • Tokuta Yokohata
}

Published online: 27 August 2014

(C) Springer-Verlag Berlin Heidelberg 2014

\section{Erratum to: Clim Dyn}

DOI 10.1007/s00382-014-2174-9

Unfortunately, the author(s) cited the work of Durack et al. incorrectly in the original paper. They stated that Durack et al. found that salinity observations suggest that global mean precipitation has increased by $8 \pm 5 \% / \mathrm{K}$ since 1950 . This is incorrect. In fact, Durack et al. found that the salinity observations suggest that the global spatial pattern of precipitation minus evaporation has intensified by $8 \pm 5 \% / \mathrm{K}$. The correction explains this error. The results and conclusions of the original paper are unaffected by the mistake.

The online version of the original article can be found under doi:10.1007/s00382-014-2174-9.

\section{F. H. Lambert $(\triangle)$}

College of Engineering, Mathematics and Physical Sciences, University of Exeter, Harrison Building, North Park Road,

Exeter EX4 4QF, UK

e-mail: f.h.lambert@exeter.ac.uk

M. J. Webb

Met Office Hadley Centre, Fitzroy Road, Exeter EX1 3PB, UK

M. Yoshimori

Atmosphere and Ocean Research Institute, The University

of Tokyo, 5-1-5, Kashiwanoha, Kashiwa, Chiba 277-8568, Japan

Present Address:

M. Yoshimori

Faculty of Environmental Earth Science, Hokkaido University,

Kita-10, Nishi-5, Kita-ku, Sapporo 060-0810, Japan

T. Yokohata

National Institute for Environmental Studies,

Tsukuba, Ibaraki 305-8506, Japan 